\title{
Highly conductive and stretchable conductors fabricated from bacterial cellulose
}

\author{
Hai-Wei Liang, Qing-Fang Guan, Zhu-Zhu, Lu-Ting Song, Hong-Bin Yao, Xuan Lei and Shu-Hong Yu
}

\begin{abstract}
Advanced materials that can remain electrically conductive under substantial elastic stretch and bending have attracted extensive interest recently owing to their broad application potentials, particularly for flexible electronics. Here, we have developed a simple and inexpensive method to fabricate highly conductive and stretchable composites using bacterial cellulose (BC) pellicles as starting materials, which can be produced in large amounts on an industrial scale via a microbial fermentation process. The prepared pyrolyzed BC (p-BC)/polydimethylsiloxane (PDMS) composites exhibit a high electrical conductivity of $0.20-0.41 \mathrm{~S} \mathrm{~cm}^{-1}$, which is much higher than conventional carbon nanotubes and graphene-based composites. More importantly, the p-BC/PDMS composites that combine high stretchability with high conductivity show great electromechanical stability. Even after 1000 stretching cycles at the maximum strain of $80 \%$, the resistance of the composites increased by only $\sim 10 \%$. The resistance increased slightly ( 4\%) after 5000 bending cycles with a maximum bending radius of $1.0 \mathrm{~mm}$. NPG Asia Materials (2012) 4, e19; doi:10.1038/am.2012.34; published online 1 June 2012
\end{abstract}

Keywords: aerogels; bacterial cellulose; carbon nanofibers; electrical conductivity; pyrolysis; stretchable conductors

\section{INTRODUCTION}

Although they possess high conductivity, conventional metal conductors cannot meet the demands for some emerging applications, particularly for flexible electronic devices, ${ }^{1-3}$ actuators, ${ }^{4}$ sensors ${ }^{5,6}$ and loudspeakers. ${ }^{7}$ These limitations have motivated intensive efforts to develop advanced electrically conductive materials capable of substantial elastic stretching and bending. ${ }^{2,8-11}$ One method for fabricating stretchable conductors is to form one dimensional 'wavy' inorganic ribbons or two dimensional 'wavy' membranes by releasing pre-strained elastomeric substrates with conductive materials lying on them. ${ }^{9} 12-16$ Such wavy conductive structures have exhibited excellent conductivity and mechanical stretchability. Another method is to embed or bond rigid and active electronic components into a soft rubbery polymer. ${ }^{2,10,17-20}$ For example, Shin et al. ${ }^{17}$ fabricated elastomeric conductive composites by the infiltration of multiwalled carbon nanotube forests with a polyurethane binder. Someya's group developed highly conductive, printable and stretchable composite films using ionic liquid, fluorinated copolymer and ultralong single-walled carbon nanotubes (SWNTs). ${ }^{1,2}$ Transparent stretchable conductors have also been fabricated by embedding well-aligned carbon nanotube (CNT) ribbons or graphene films in polydimethylsiloxane (PDMS). ${ }^{11,19}$ Although mechanical stretchability was successfully achieved, most of the above studies demonstrated that the resistance of the obtained composites increases rapidly with tensile strain $\left(>100 \%\right.$ increase of resistance at $100 \%$ strain). ${ }^{5,10,11,17,18}$
Very recently, a new strategy was developed for generating stretchable conductors by backfilling an infinite connected network of conducting fillers (such as single-walled carbon nanotubesaerogels $^{21}$ or graphene foams ${ }^{22}$ ) with an elastic polymer. Owing to the prefabricated three dimensional (3D) network structures, these composites showed a relatively high electrical conductivity and electromechanical stability under stretching and bending. ${ }^{21,22}$ However, the cost of single-walled carbon nanotubes and graphene fabricated from the chemical vapor deposition process is still high. It is quite desirable to explore inexpensive, green and scalable processes to lower the fabrication cost for industrial applications.

Bacterial cellulose (BC) has long been used as the raw material of nata-de-coco, an indigenous dessert food of the Philippines, for which one centimeter thick gel sheets fermented with coconut-water are cut into cubes and immersed in sugar sirup. ${ }^{23} \mathrm{BC}$ can be produced in large amounts on an industrial scale via a microbial fermentation process. $^{23,24}$ It is well known that BC pellicles are composed of interconnected 3D networks of nanofibers that have a native cellulose I crystal structure with high-molar-mass, hydrogen-bonded polymer chains in an extended-chain conformation. ${ }^{23,25}$ In addition, BC has been used as a precursor material to prepare graphitized films ${ }^{26}$ and carbon nanofibers. ${ }^{27}$ Inspired by this biomaterial, ${ }^{28,29}$ we propose to construct 3D conductive carbon nanofiber networks from BC pellicles for fabricating stretchable conductors. The major advantages of using $\mathrm{BC}$ as a precursor material include easy fabrication, low cost and its

Division of Nanomaterials and Chemistry, Hefei National Laboratory for Physical Sciences at the Microscale (HFNL), Department of Chemistry, The National Synchrotron Radiation Laboratory, University of Science and Technology of China (USTC), Hefei, Anhui, PR China

Correspondence: Professor S-H Yu, Division of Nanomaterials and Chemistry, HFNL, Department of Chemistry, The National Synchrotron Radiation Laboratory, USTC, Jinzhai Road 96, Hefei 230026, PR China.

E-mail: shyu@ustc.edu.cn

Received 23 March 2012; revised 25 April 2012; accepted 30 April 2012 
mechanically robust $3 \mathrm{D}$ network structures, which is a key point for the electromechanical performance of the final composites. In this paper, we demonstrate that $\mathrm{BC}$ pellicles can be employed as starting materials for fabricating stretchable conductors. The prepared pyrolyzed BC (p-BC)/PDMS composites exhibit a high conductivity and extraordinary electromechanical stability even under high stretching and bending strain.

\section{MATERIALS AND METHODS}

\section{Materials}

Purified BC pellicles with fiber content of $\sim 1 \%(\mathrm{vol} / \mathrm{vol})$ were kindly provided by Ms CY Zhong (Hainan Yeguo Foods Co., Ltd., Hainan, China). The fibers were produced by the bacterial strain Acetobacter xylinum using a culture of coconut milk and sucrose. ${ }^{24}$ Sylgard 184 silicone elastomer base and curing agent were purchased from Dow Corning (Midland, MI, USA).

\section{Methods}

The $\mathrm{BC}$ pellicles were first cut into rectangular or cubic shapes with a sharp blade, frozen in liquid nitrogen $\left(-196^{\circ} \mathrm{C}\right)$ and then freeze-dried in a bulk tray dryer (Labconco Corporation, Kansas City, MO, USA) at a sublimating temperature of $-48^{\circ} \mathrm{C}$ and a pressure of $0.04 \mathrm{mbar}$. The obtained $\mathrm{BC}$ aerogels were then pyrolyzed under flowing argon at $600-1450{ }^{\circ} \mathrm{C}$ to generate black and ultralight $\mathrm{p}-\mathrm{BC}$ aerogels. The $\mathrm{p}-\mathrm{BC} / \mathrm{PDMS}$ composites were fabricated by infiltrating the $\mathrm{p}-\mathrm{BC}$ aerogels with PDMS pre-polymer, a viscous mixture of base/curing agent (Sylgard 184, Dow Corning), followed by degassing in a vacuum oven for $2 \mathrm{~h}$ and thermally curing at $70^{\circ} \mathrm{C}$ for $1 \mathrm{~h}$.

\section{Characterization}

The electrical conductivity of $\mathrm{p}-\mathrm{BC}$ aerogels and $\mathrm{p}-\mathrm{BC} / \mathrm{PDMS}$ composites was measured by a two-probe method with the PM5 Analytical Probe System (Cascade Microtech, Inc., Beaverton, OR, USA) and Keithley 4200 SCS at room temperature in air. Copper wires were embedded and connected to the p-BC aerogels with silver paste (Dupont 4929N, DuPont Corporation, Wilmington, DE, USA) before infiltration with PDMS pre-polymer. The compressive tests of the $\mathrm{BC}$ and $\mathrm{p}-\mathrm{BC}$ aerogels were performed by using an Instron 5565A, equipped with two flat-surface compression stages and $500 \mathrm{~N}$ load cells. The strain ramp rate was maintained at $10 \mathrm{~mm}$ per min for all of the tests. The sample size was measured using a standard caliper. Scanning electron microscopy was performed with a field emission scanning electron microanalyzer (Zeiss Supra 40, Carl Zeiss AG, Baden-Wurttemberg, Oberkochen, Germany) after coating the samples with an Au layer of approximately $3 \mathrm{~nm}$ using a Sputter Coater (Model BAL-TEC SCD 005).

\section{RESULTS AND DISCUSSION}

Figure 1a describes the synthetic pathway. A piece of purified BC pellicle with the size of $200 \times 230 \times 4 \mathrm{~mm}^{3}$ was first cut into a rectangular shape with a sharp blade (step 1). To prevent the collapse of the gel network, a freeze-drying technique was used to remove the water from the small piece of $\mathrm{BC}$ pellicle to form a porous cellulose nanofibrous aerogel (step 2). The dried $\mathrm{BC}$ aerogel was then pyrolyzed under flowing argon at $600-1450{ }^{\circ} \mathrm{C}$ to generate a black, ultralight and conductive aerogel (step 3). For measuring the mechanical properties of aerogels, another BC pellicle with a thickness of $12 \mathrm{~mm}$ was cut into a cubic shape, freeze-dried and pyrolyzed a

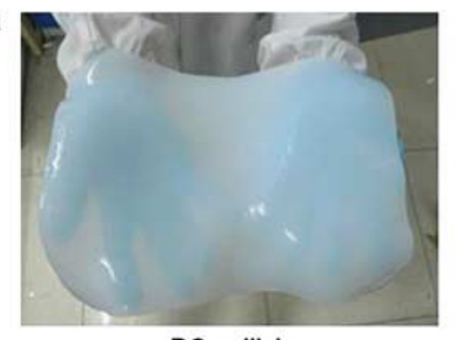

$\mathrm{BC}$ pellicle

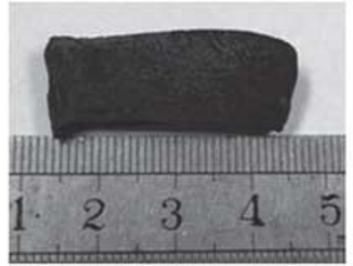

p-BC aeroge

b

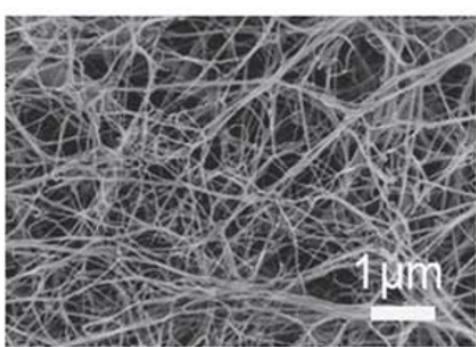

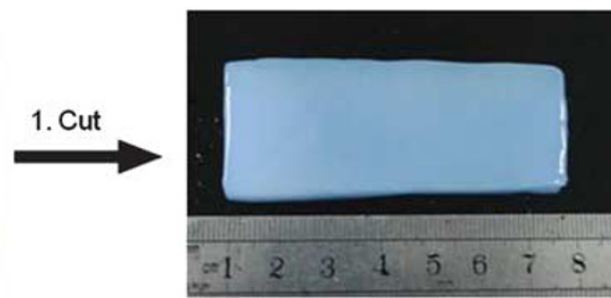

2. Freeze drying

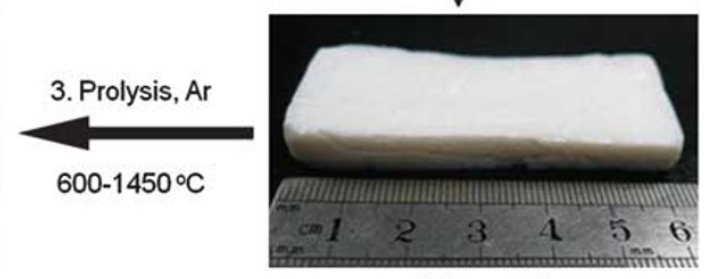

$\mathrm{BC}$ aerogel

C

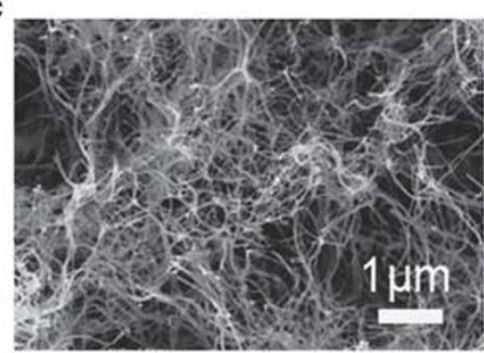

Figure 1. (a) The fabrication process of BC-based materials. (1) A large-sized BC pellicle $\left(200 \times 230 \times 3 \mathrm{~mm} \mathrm{~m}^{3}\right)$ was cut into the desired shape with a sharp blade. (2) After freeze-drying of the wet BC pellicles, the ultralight BC aerogel was obtained. (3) Further pyrolysis treatment of the BC aerogel under flowing argon produced the black p-BC aerogel. (b, c) Scanning electron microscopy images of the BC and p-BC aerogels, respectively. 
(Supplementary Figure S1). The apparent density of the dried BC aerogels was $\sim 7 \mathrm{mg} \mathrm{cm}^{-3}$. The porosity was estimated to be $99.56 \%$ based on calculations using a density of $1.6 \mathrm{~g} \mathrm{~cm}^{-3}$ for the cellulose nanofibrils. ${ }^{25}$ During the pyrolysis process, the BC sample gradually shrank to half of its original size in three dimensions, whereas the volume shrank by $\sim 88 \%$. The obtained p-BC aerogels are extremely light, and have an ultralow density of $3-4 \mathrm{mg} \mathrm{cm}^{-3}$ with a high porosity of $>99.7 \%$. Scanning electron microscopy images of the freeze-dried $\mathrm{BC}$ aerogel reveal a highly porous network structure consisting of numerous nanofibers (Figure 1b). High-magnification scanning electron microscopy observation indicates that these nanofibers with a diameter of $20-50 \mathrm{~nm}$ are highly interconnected with large numbers of junctions (Supplementary Figure S2). ${ }^{25}$ After a pyrolysis treatment, the $\mathrm{p}-\mathrm{BC}$ aerogels obtain a $3 \mathrm{D}$ network structure, except that the fiber diameter is decreased to $10-20 \mathrm{~nm}$ (Figure 1c) due to the carbonization of the $\mathrm{BC}$ and evaporation of volatile species such as $\mathrm{CO}, \mathrm{CO}_{2}$, methanol, and acetic acid during pyrolysis. ${ }^{27} \mathrm{We}$ propose that this unique $3 \mathrm{D}$ network structure is responsible for the outstanding mechanical and conducting properties of the p-BC aerogels (see below for details).

Unlike conventional low-density aerogels that are brittle and tend to break even upon a small compression, the present $\mathrm{BC}$ and $\mathrm{p}-\mathrm{BC}$ aerogels composed of interconnected nanofibers exhibited high flexibility, that is, suppression of brittleness. The compressive properties of the BC-based aerogels were studied quantitatively, and the results are shown in Figures $2 \mathrm{a}$ and $\mathrm{b}$. The $\mathrm{BC}$ aerogels can be compressed without disintegration after being subject to a maximum strain of $80 \%$ with a compressive strength of $18.0 \mathrm{kPa}$ (Figure 2a). When the compressive load was removed, the $\mathrm{BC}$ aerogel did not return to its original shape because of the strongly interacting surface hydroxyl groups in the $\mathrm{BC}$ nanofibers (the inset in Figure 2a). In contrast, the $\mathrm{p}-\mathrm{BC}$ aerogel showed a highly elastic compressibility and almost completely recovered its original volume after the compression was released (Figure $2 \mathrm{~b}$ and inset), although it had a much lower compressive strength of $2.5 \mathrm{kPa}$ at $80 \%$ strain, most likely due to the reduced density after pyrolysis treatment. The $\mathrm{p}-\mathrm{BC}$ aerogel was subjected to a fatigue cyclic compression test $(\varepsilon=80 \%)$ by undergoing 100 loading/unloading cycles (Figure $2 \mathrm{~b}$ ). The unloading curves show that the stresses remained above zero until $\varepsilon=0$, suggesting almost complete recovery of the compressed aerogel and no thickness reduction after the fatigue test. The degradation at the maximum compressive strength (at $80 \%$ strain) of the p-BC aerogel was only $7.5 \%$ after 100 cycles. It is believed that the robust mechanical properties of the $\mathrm{p}-\mathrm{BC}$ aerogel are attributed to its unique interconnected 3D network structures and elimination of the hydrogenbond interaction during pyrolysis.

The flexible $\mathrm{p}-\mathrm{BC} / \mathrm{PDMS}$ composites were prepared by infiltrating the $\mathrm{p}-\mathrm{BC}$ aerogels with the polymer resin (Dow Corning Sylgard 184) under vacuum before curing at $70{ }^{\circ} \mathrm{C}$ (the inset in Figure $2 \mathrm{c}$ ). The dimensions of the aerogels remained unchanged after infiltrating and curing. Scanning electron microscopy images of the fracture surface of the composites show that the carbon nanofibers are homogenously distributed throughout the polymer matrix (Figure $2 \mathrm{c}$ and Supplementary Figure S3). The electric resistance of the p-BC aerogels and their composites with PDMS were measured by the two-probe method. All of the measured current-voltage (I-V) curves showed a linear dependence, indicating good ohmic contacts between the composites and the electrodes (Supplementary Figure S4). The results

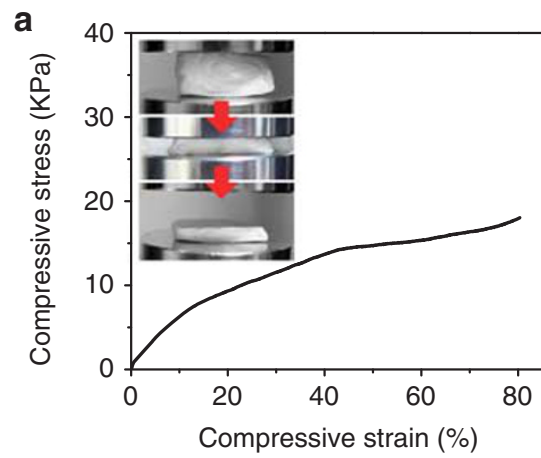

b

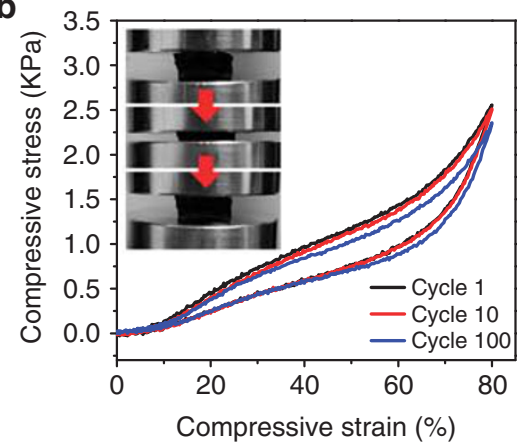

C
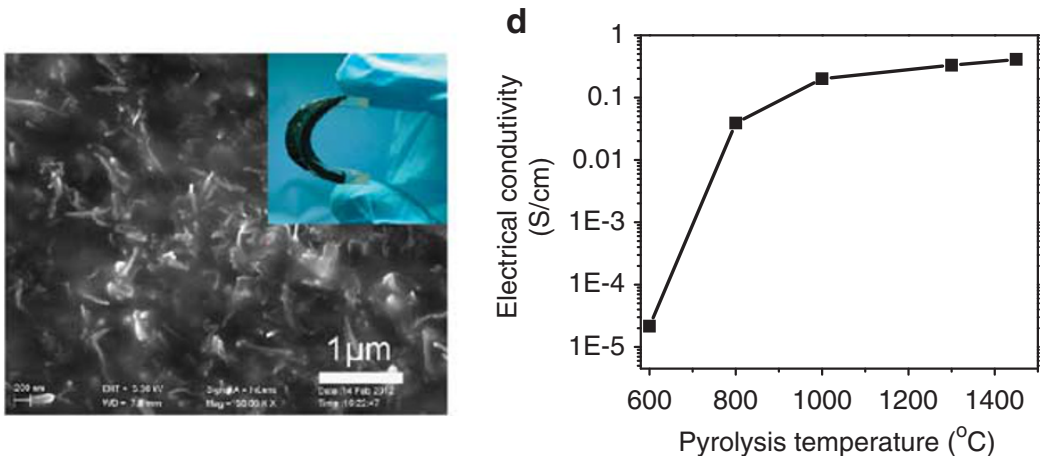

Figure 2. (a) Compressive stress-strain curve of the $B C$ aerogel at a set strain of $80 \%$. (b) Cyclic stress-strain curves of the $p$ - $B C$ aerogel at a maximum strain of $80 \%$, showing a very small permanent deformation after 100 compression cycles. The insets in (a) and (b) show the sequential photographs of the $\mathrm{BC}$ and $\mathrm{p}-\mathrm{BC}$ aerogels during the compression process, illustrating their different mechanical properties. (c) Scanning electron microscopy image of the fracture surface of the typical p-BC/PDMS composite. The inset shows the photograph of a bent composite, showing its flexibility. (d) Electrical conductivity of $\mathrm{p}-\mathrm{BC} / \mathrm{PDMS}$ composites as a function of the pyrolysis temperature. 
show that the electrical conductivity of the $\mathrm{p}-\mathrm{BC} / \mathrm{PDMS}$ composites was approximately equal to that of the initial p-BC aerogels (Supplementary Figure S4), indicating that introduction of the PDMS matrix does not damage the interconnected 3D network of carbon nanofibers. In addition, the electrical conductivity of the p-BC aerogels and $\mathrm{p}-\mathrm{BC} / \mathrm{PDMS}$ composites increases rapidly with the pyrolysis temperature (Figure $2 \mathrm{~d}$ ) because higher temperature results in carbon nanofibers with a higher degree of graphitization. ${ }^{27}$ The composites show an electrical conductivity of $>0.2 \mathrm{~S} \mathrm{~cm}^{-1}$ when the pyrolysis temperature is $>1000{ }^{\circ} \mathrm{C}$ despite the very low loading of the active electronic component ( $<0.3 \mathrm{vol} \%)$. The electrical conductivity of the composite made from the $\mathrm{BC}$ aerogel that was pyrolyzed at $1450{ }^{\circ} \mathrm{C}$ reached $0.41 \mathrm{~S} \mathrm{~cm}^{-1}$, which is much higher than traditional CNTs and graphene-based composites prepared by solution mixing with such a low filler content (Supplementary Table S1). For the latter, electronically active fillers, such as CNTs and graphene nanosheets, are embedded separately in the polymer matrix, and the electrical conductivity of these composites is strongly dependent on the electron percolation between the filler particles. ${ }^{30,31}$ In contrast, in our composites, because the infinite interconnected network of conductive carbon nanofibers remained unchanged even after infiltrating with an insulating polymer, electrons can move very quickly through the network it, resulting in a higher electrical conductivity. When compared with the present method, the solution mixing method exhibits more versatility, such as allowing for easy control of the filler content, but the electrical conductivity of the composite obtained by solution mixing can only be controlled in a limited range because high filler content likely results in phase disengagement and fails to form mechanically robust composites. We also measured the stress-strain curves of the p-BC/PDMS composites and pure PDMS, and the results are shown in Supplementary Figure S5. The fabricated p-BC/PDMS composite exhibited high stretchability with a strain-to-failure rate of $118 \%$, which is somewhat lower than that of the pure PDMS sheet $(\sim 150 \%)$.

The extraordinary electrical conductivity of the p-BC/PDMS composites, as well as their good mechanical properties, indicates a great potential as flexible and stretchable conductors (Figure 3). We evaluated the stretchability and foldability of the composite conductors by measuring resistances with respect to stretching strain and bending radii, which were carried out with a high-precision mechanical system (Instron 5565A). The composite fabricated from the BC aerogel that was pyrolyzed at $1300^{\circ} \mathrm{C}$ was tested and had an initial conductivity of $0.33 \mathrm{~S} \mathrm{~cm}^{-1}$. The composites remained robust after repeated stretching-releasing cycles and bending.

We first investigated the resistance variation as a function of uniaxial tensile strain from 0 to $80 \%$ (Figures $3 \mathrm{a}$ and b). Note that the composite remained conductive until it broke at $118 \%$ strain (Supplementary Figure S6). During the first cycle of stretchingreleasing, the resistance of $\mathrm{p}$-BC/PDMS composite increased nearly linearly with increasing tensile strain (Figure 3a). The resistance increase $\left(\Delta R / R_{0}\right)$ at the maximum strain $(80 \%)$ is $14.8 \%$, which is much lower than the reported values for conventional graphene- and CNT-based stretchable conductors ${ }^{2,10,11,18}$ and is comparable to that of emerging single-walled carbon nanotubes/PDMS ${ }^{21}$ and graphene foam/PDMS ${ }^{22}$ composites. When the stretched composite was released to the unstrained state, we observed $9.2 \%$ loss in conductivity, implying a partial breaking or cracking of the nanofibrous network. We then performed a second stretching cycle,
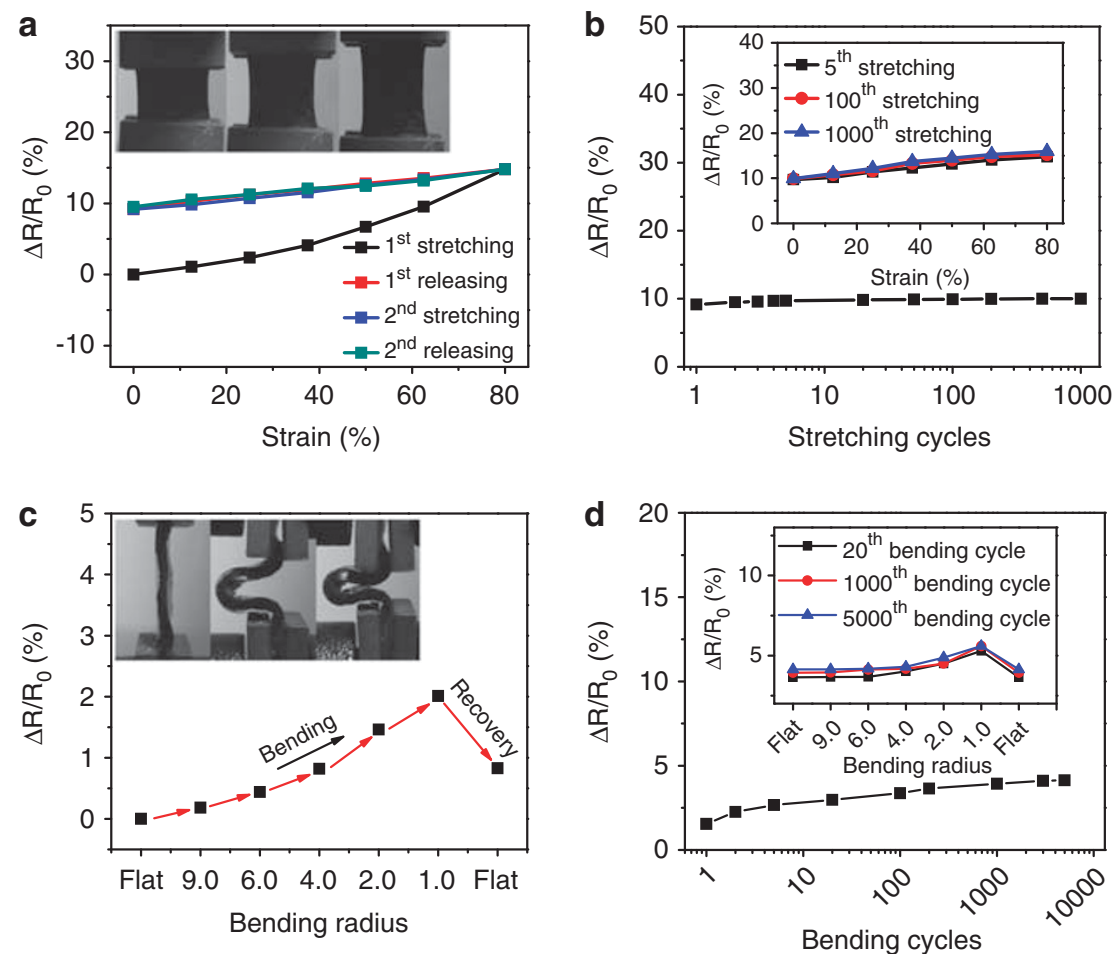

Figure 3 Resistance change of the p-BC/PDMS composite under mechanical deformations. (a) Variation of the normalized resistance ( $\Delta R / R_{0}$ ) of the composite as a function of tensile strain up to $80 \%$ in the first two stretch-release cycles. The inset shows the stretching process. (b) $\Delta R / R_{0}$ as a function of stretching cycles at a strain up to $80 \%$. The inset shows the fifth, hundredth, thousandth stretching cycles. (c) $\Delta R / R_{0}$ of the composite at a bend radius of up to $1.0 \mathrm{~mm}$ in the first bending cycle. The inset shows the bending process. (d) $\Delta R / R_{0}$ as a function of the bend cycles at a maximum bend radius of $1.0 \mathrm{~mm}$. The inset shows the twentieth, thousandth, fivethousandth bending cycles. $R_{0}$ refers to the electrical resistance of the pristine composite, and $\Delta R$ refers to the resistance change of the bent or stretched composite relative to that of the pristine composite. 
where the $\Delta R / R_{0}$ at the maximum strain was the same as that during the first cycle, and only another $0.3 \%$ loss in conductivity was observed when the strain was fully released (Figure 3a). In fact, except for the first cycle, the resistance showed no obvious change between each stretching-releasing cycle. The p-BC/PDMS composite reached a stable $\Delta R / R_{0}$ after five stretching-releasing cycles and showed great reversibility thereafter (the inset in Figure $3 \mathrm{~b}$ ). It is worth mentioning that there was only $0.8 \%$ increase in the $\Delta R / R_{0}$ from the first to the thousandth cycle (Figure $3 \mathrm{~b}$ ), which shows the excellent electromechanical properties of the present composites.

We also investigated the resistance variation under bending deformations, as shown in Figures $3 \mathrm{c}$ and $\mathrm{d}$. During the first bending cycle, the resistance showed a slight increase of $2.0 \%$ at a bending radius of $1.0 \mathrm{~mm}$ and could recover partially after unbending (Figure 3c), similar to the results in the stretching tests. As the number of bending cycles increased, the resistance variation of composites gradually became stable, and the resistance increased only by $4.1 \%$ even after 5000 cycles for a bend radius of $1.0 \mathrm{~mm}$ (Figure $3 \mathrm{~b}$ and inset).

A simple device was demonstrated for lighting a commercial LED using the $\mathrm{p}-\mathrm{BC} / \mathrm{PDMS}$ composites. As shown in Supplementary Figure S7, the brightness of the LED did not change significantly when the $\mathrm{p}$-BC/PDMS composite was twisted or stretched. These results show the extraordinary electromechanical stability of $\mathrm{p}-\mathrm{BC} /$ PDMS composites and their great potential for high-performance stretchable and flexible conductors. We suspect that the outstanding electromechanical properties of the composites result from the $3 \mathrm{D}$ network structures of the $\mathrm{p}-\mathrm{BC}$ aerogels and their robust mechanical properties, as revealed in Figure $2 \mathrm{~b}$. Because the robust interconnected $3 \mathrm{D}$ network of carbon nanofibers was preformed and remained unchanged after infiltrating with PDMS, the conductive nanofibers within the composite could slide past one other without losing junction points while being supported by the PDMS during stretching and bending; thus little variation in conductivity was observed.

\section{CONCULUSIONS}

In summary, we have developed a new type of highly conductive and stretchable conductors using an inexpensive and green material, BC pellicles, as a precursor, which can be produced in large amounts on an industrial scale via a microbial fermentation process. The prepared pyrolyzed p-BC/PDMS composites with a high electrical conductivity of $0.20-0.41 \mathrm{~S} \mathrm{~cm}^{-1}$ exhibit extraordinary electromechanical stability even under high tensile and bending strain. It is believed that the outstanding electromechanical properties of the composites are due to the robust and preformed 3D networks of the $\mathrm{p}-\mathrm{BC}$ aerogels that provide an interconnected pathway through which electrons can quickly move. Because of the advantages of the present method, such as inexpensive and accessible precursor material and easy fabrication, it is possible to scale up the fabrication process for industrial applications in large-scale flexible, stretchable and foldable electronics.

\section{CONFLICT OF INTEREST}

The authors declare no conflicts of interest.

\section{ACKNOWLEDGEMENTS}

SHY acknowledge special funding support from the National Basic Research Program of China (Grant 2010CB934700), the National Natural Science Foundation of China (Grants 91022032, 21061160492, J1030412), the Chinese Academy of Sciences (Grant KJZD-EW-M01-1), the International Science \& Technology Cooperation Program of China (Grant 2010DFA41170), and the Principal Investigator Award by the National Synchrotron Radiation
Laboratory at the USTC. HWL acknowledge Fundamental Research Funds for the Central Universities.

Author contributions: SHY conceived the project and planned the experiments. HWL and QFG prepared samples and carried out the characterizations. SHY and HWL analyzed the data and wrote the paper. All authors discussed the results and commented on the paper.

1 Sekitani, T., Nakajima, H., Maeda, H., Fukushima, T., Aida, T., Hata, K. \& Someya, T. Stretchable active-matrix organic light-emitting diode display using printable elastic conductors. Nat. Mater. 8, 494-499 (2009).

2 Sekitani, T., Noguchi, Y., Hata, K., Fukushima, T., Aida, T. \& Someya, T. A rubberlike stretchable active matrix using elastic conductors. Science 321, 1468-1472 (2008).

3 Pang, S., Hernandez, Y., Feng, X. \& Müllen, K. Graphene as transparent electrode material for organic electronics. Adv. Mater. 23, 2779-2795 (2011).

4 Koerner, H., Price, G., Pearce, N. A., Alexander, M. \& Vaia, R. A. Remotely actuated polymer nanocomposites - stress-recovery of carbon-nanotube-filled thermoplastic elastomers. Nat. Mater. 3, 115-120 (2004).

5 Lipomi, D. J., Vosgueritchian, M., Tee, B. C. K., Hellstrom, S. L., Lee, J. A., Fox, C. H. \& Bao, Z. Skin-like pressure and strain sensors based on transparent elastic films of carbon nanotubes. Nat. Nanotechnol. 6, 788-792 (2011).

6 Someya, T., Sekitani, T., Iba, S., Kato, Y., Kawaguchi, H. \& Sakurai, T. A large-area, flexible pressure sensor matrix with organic field-effect transistors for artificial skin applications. Proc. Natl Acad. Sci. USA 101, 9966-9970 (2004).

7 Xiao, L., Chen, Z., Feng, C., Liu, L., Bai, Z.-Q., Wang, Y., Qian, L., Zhang, Y., Li, Q., Jiang, K. \& Fan, S. Flexible, stretchable, transparent carbon nanotube thin film loudspeakers. Nano Lett. 8, 4539-4545 (2008).

8 Rogers, J. A., Someya, T. \& Huang, Y. Materials and mechanics for stretchable electronics. Science 327, 1603-1607 (2010).

9 Khang, D.-Y., Jiang, H., Huang, Y. \& Rogers, J. A. A stretchable form of singlecrystal silicon for high-performance electronics on rubber substrates. Science $\mathbf{3 1 1}$, 208-212 (2006).

10 Chun, K. Y., Oh, Y., Rho, J., Ahn, J. H., Kim, Y. J., Choi, H. R. \& Baik, S. Highly conductive, printable and stretchable composite films of carbon nanotubes and silver. Nat. Nanotechnol 5, 853-857 (2010).

11 Kim, K. S., Zhao, Y., Jang, H., Lee, S. Y., Kim, J. M., Ahn, J. H., Kim, P., Choi, J. Y. \& Hong, B. H. Large-scale pattern growth of graphene films for stretchable transparent electrodes. Nature 457, 706-710 (2009).

12 Bowden, N., Brittain, S., Evans, A. G., Hutchinson, J. W. \& Whitesides, G. M. Spontaneous formation of ordered structures in thin films of metals supported on an elastomeric polymer. Nature 393, 146-149 (1998).

13 Lacour, S. P., Wagner, S., Huang, Z. Y. \& Suo, Z. Stretchable gold conductors on elastomeric substrates. Appl. Phys. Lett. 82, 2404-2406 (2003).

$14 \mathrm{Kim}$, D.-H., Xiao, J., Song, J., Huang, Y. \& Rogers, J. A. Stretchable, curvilinear electronics based on inorganic materials. Adv. Mater. 22, 2108-2124 (2010).

15 Wang, X. L., Hu, H., Shen, Y. D., Zhou, X. C. \& Zheng, Z. J. Stretchable conductors with ultrahigh tensile strain and stable metallic conductance enabled by prestrained polyelectrolyte nanoplatforms. Adv. Mater. 23, 3090-3094 (2011).

16 Zhu, Y. \& Xu, F. Buckling of aligned carbon nanotubes as stretchable conductors: a new manufacturing strategy. Adv. Mater. 24, 1073-1077 (2012).

17 Shin, M. K., Oh, J., Lima, M., Kozlov, M. E., Kim, S. J. \& Baughman, R. H. Elastomeric conductive composites based on carbon nanotube forests. Adv. Mater. 22, 2663-2667 (2010).

18 Yu, Z., Niu, X., Liu, Z. \& Pei, Q. Intrinsically stretchable polymer light-emitting devices using carbon nanotube-polymer composite electrodes. Adv. Mater. 23, 3989-3994 (2011).

19 Zhang, Y., Sheehan, C. J., Zhai, J., Zou, G., Luo, H., Xiong, J., Zhu, Y. T. \& Jia, Q. X. Polymer-embedded carbon nanotube ribbons for stretchable conductors. Adv. Mater. 22, 3027-3031 (2010).

20 Jung, Y. J., Kar, S., Talapatra, S., Soldano, C., Viswanathan, G., Li, X., Yao, Z., Ou, F. S., Avadhanula, A., Vajtai, R., Curran, S., Nalamasu, O. \& Ajayan, P. M. Aligned Carbon nanotube-polymer hybrid architectures for diverse flexible electronic applications. Nano Lett. 6, 413-418 (2006).

$21 \mathrm{Kim}$, K. H., Vural, M. \& Islam, M. F. Single-walled carbon nanotube aerogel-based elastic conductors. Adv. Mater. 23, 2865-2869 (2011).

22 Chen, Z., Ren, W., Gao, L., Liu, B., Pei, S. \& Cheng, H.-M. Three-dimensional flexible and conductive interconnected graphene networks grown by chemical vapour deposition. Nat. Mater. 10, 424-428 (2011).

23 Iguchi, M., Yamanaka, S. \& Budhiono, A. Bacterial cellulose-a masterpiece of nature's arts. J. Mater. Sci. 35, 261-270 (2000).

24 China Patent. A microbial fermentation process for fabricating dietary fiber by using coconut water as raw materials, ZL96100534.3 (2001).

25 Yano, H., Sugiyama, J., Nakagaito, A. N., Nogi, M., Matsuura, T., Hikita, M. \& Handa, K. Optically transparent composites reinforced with networks of bacterial nanofibers. Adv. Mater. 17, 153-155 (2005).

26 Yoshino, K., Matsuoka, R., Nogami, K., Yamanaka, S., Watanabe, K., Takahashi, M. \& Honma, M. Graphite film prepared by pyrolysis of bacterial cellulose. J. Appl. Phys. 68, 1720-1725 (1990).

27 Wan, Y. Z., Zuo, G. F., Yu, F., Huang, Y. A., Ren, K. J. \& Luo, H. L. Preparation and mineralization of three-dimensional carbon nanofibers from bacterial cellulose as 
potential scaffolds for bone tissue engineering. Surf. Coat. Technol. 205, 2938-2946 (2011).

28 Wang, J., Cheng, Q. \& Tang, Z. Layered nanocomposites inspired by the structure and mechanical properties of nacre. Chem. Soc. Rev. 41, 1111-1129 (2012).

29 Cheng, Q., Li, M., Jiang, L. \& Tang, Z. Bioinspired layered composites based on flattened double-walled carbon nanotubes. Adv. Mater. 24, 1838-1843 (2012).

30 Kuilla, T., Bhadra, S., Yao, D. H., Kim, N. H., Bose, S. \& Lee, J. H. Recent advances in graphene based polymer composites. Prog. Polym. Sci. 35, 1350-1375 (2010).
31 Spitalsky, Z., Tasis, D., Papagelis, K. \& Galiotis, C. Carbon nanotube-polymer composites: chemistry, processing, mechanical and electrical properties. Prog. Polym. Sci. 35, 357-401 (2010).

(c) This work is licensed under the Creative Commons Attribution-NonCommercial-No Derivative Works 3.0 Unported License. To view a copy of this license, visit http:// creativecommons.org/licenses/by-nc-nd/3.0/

Supplementary Information accompanies the paper on the NPG Asia Materials website (http://www.nature.com/am) 